\title{
LA CIRCULACIÓN DE LAS IDEAS POSITIVISTAS EN ARGENTINA Y EN MÉXICO: EDITORES Y TRADUCTORES (1850-1950)
}

\author{
Nayelli Castro \\ ncast016@uottawa.ca \\ Clara Foz \\ cfoz@uottawa.ca \\ Universidad de Ottawa
}

\section{Resumen}

La historiografía sobre el tema ha mostrado que la introducción de la filosofía de Auguste Comte en Latinoamérica dio lugar a un positivismo profundamente heterogéneo. Su combinación con los escritos de Herbert Spencer, Charles Darwin y John Stuart Mill, la circulación de impresos, tanto en lengua original como en traducción, y el desarrollo de un mercado editorial internacional a fines del siglo XIX son algunos de los factores que contribuyen a explicar dicha heterogeneidad. El presente trabajo estudia la circulación de las ideas positivistas en Argentina y en México desde la perspectiva de los editores y traductores activos entre 1850 y 1950, un periodo clave para la organización política de los nuevos Estados latinoamericanos y para la constitución de sus instituciones educativas.

\begin{abstract}
"Positivism on the Move: Translators and Publishers in Mexico and Argentina (1850-1950)"

Historians have demonstrated that after its introduction to Latin America, Auguste Comte's philosophy became an eclectic Positivism. Its combination with the writings of Herbert Spencer, Charles Darwin, and John Stuart Mill, the circulation of printed materials in their original language and in translation, as well as the development of an international publishing market in the late $19^{\text {th }}$ century are some of the factors that
\end{abstract}


contribute to explain this eclecticism. This paper studies the flow of Positivist ideas in Mexico and Argentina from the perspective of its publishers and translators, in a period that is fundamental for the organization of the new Latin American States and their educational policies (1850-1950).

Keywords: Positivism. Translators. Publishers. Agency. History of translation

Palabras clave: Positivismo. Traductores. Editores. Agentes. Historia de la traducción

Manuscript received on June 30, 2012 and accepted on July 25, 2012. 


\section{Los positivismos latinoamericanos: Argentina y México}

Auguste Comte (1798-1857), personaje central del positivismo decimonónico, publica los seis tomos de su Cours de philosophie positive entre 1830 y 1842. La repercusión de esta obra en el contexto europeo del siglo XIX y en el de los recién formados Estados latinoamericanos no es menor. De acuerdo con Zea, "el positivismo se presentará en Latinoamérica como una tabla de salvación en la violenta tempestad que siguiera al logro de la emancipación política alcanzada frente a la colonización ibera" (1980: xxvi). No obstante, el positivismo latinoamericano no puede considerarse como una reproducción del europeo. Así, para Ardao (1963: 516), mientras en Europa el positivismo se desarrolló en una dinámica de "influencias recíprocas", en Latinoamérica, las diferentes manifestaciones del positivismo evolucionaron de manera paralela. De la misma manera, mientras que en Europa las ideas positivistas resultaron de la institucionalización de las ciencias, en Latinoamérica, estas ideas dieron lugar al desarrollo de un pensamiento científico. Aún más, los intelectuales latinoamericanos decimonónicos combinaron las ideas comtianas con el utilitarismo de Jeremy Bentham (1748-1832) y de John Stuart Mill (1806-1873), el evolucionismo de Herbert Spencer (1820-1903) y de Charles Darwin (1809-1882), y, a menudo, negociaron con corrientes de pensamiento opuestas como el krausismo ${ }^{1}$ y el espiritualismo de Victor Cousin (1792-1867).

En estas páginas, nos interesa investigar la circulación de las ideas positivistas en Argentina y en México por la importancia de ambos países para la edición y distribución de impresos en la región. Aunque el español, legado del periodo colonial, permitió la circulación de originales y traducciones sin muchas dificultades y, en esa medida creó un "espacio lingüístico y de intercambios culturales internacionales" que es difícil delimitar (Sorá 2009: 95),

1. Movimiento cultural español basado en la importación de los escritos del filósofo idealista alemán K. Ch. F. Krause (1781-1832). De escasa repercusión en el ámbito filosófico alemán, su introducción en España por medio de las traducciones de Julián Sanz del Río (1814-1869) dio lugar al desarrollo de una filosofía práctica y de un pensamiento pedagógico (Sánchez Ortíz de Urbina 1966) 
por razones metodológicas hemos decidido centrar nuestro trabajo en estos dos países. Como lo muestra Pura Fernández en su estudio sobre los impresos en castellano en el siglo XIX, ambos fueron destinos importantes para la exportación de libros procedentes de Bélgica, España y Francia (1998: 174). No olvidemos, además, la importancia de las ideas positivistas para la producción científica y cultural y para la organización política y educativa de toda Hispanoamérica en este periodo.

Nuestra decisión de limitar el periodo de estudio al siglo comprendido entre 1850 y 1950 no implica que los textos de Comte, Darwin, Mill y Spencer hayan dejado de editarse o de circular después de esa fecha. Sin embargo, pensamos que fijar estos límites temporales permitirá esbozar la circulación del positivismo en el contexto señalado antes de la introducción de la fenomenología y el existencialismo, corrientes de pensamiento que ocuparán una posición predominante en la segunda mitad del siglo XX.

\subsection{Los "agentes de traducción" del positivismo}

La historiografía existente sobre el positivismo latinoamericano hace hincapié en algunos de los "personajes" clave en la introducción de las ideas positivistas en Latinoamérica. Por lo tanto, la narración de los historiadores exalta ante todo el papel desempeñado por los pioneros o "precursores", produciendo con ello relatos épicos de las naciones latinoamericanas de las que se han ocupado. Rara vez dichos relatos se refieren a la manera en que las ideas positivistas fueron difundidas y alcanzaron un estatus institucional en libros de texto, políticas educativas y debates intelectuales. Con el fin de completar la perspectiva de estas fuentes historiográficas hemos recurrido a los catálogos de las bibliotecas nacionales de Argentina y México en busca de las traducciones al español de obras de Auguste Comte, Herbert Spencer, Charles Darwin y John Stuart Mill. Este procedimiento corresponde, por un lado, a una misión arqueológica, cuyo objetivo es desentrañar una historia menos estudiada (Pym 1998: 5) y, por el otro, a una perspectiva interpretativa que, a menudo opacada en los relatos épicos nacionales, busca contribuir tanto a la historia de la traducción como a la historiografía sobre el positivismo latinoamericano.

Las obras que hemos encontrado corresponden al fondo físico de las bibliotecas consultadas y pueden haber circulado en Argentina y México entre 1850 y 1950 o haber sido integradas a esas bibliotecas posteriormente. ${ }^{2}$ Nos

2. Los datos arrojados por las búsquedas realizadas en sendos catálogos distan de ser sistemáticos. En efecto, cada biblioteca presenta diferentes formatos de ficha catalográfica 
han interesado en particular aquellas cuyo pie de imprenta, esto es, el lugar y la fecha de publicación, corresponde al contexto que hemos definido para nuestro estudio. Si bien no pretendemos haber logrado un listado exhaustivo, estas búsquedas han permitido determinar que en las traducciones que nos interesan interviene una nutrida nómina de editores y traductores congregados en torno a editoriales, imprentas o fundaciones culturales de Argentina, Brasil, Chile, España, Estados Unidos, Francia, México, Paraguay y Portugal.

Es por ello que la noción de "agentes de traducción" (Bandia y Milton 2009) se antoja especialmente pertinente. Con ella se hace referencia a los "productores de textos, mediadores que modifican un texto, o lo resumen, editan, revisan y traducen", a los "mecenas, organizadores de salones literarios, políticos y organizaciones que contribuyen a reformar políticas culturales y lingüísticas", así como también a las "revistas, diarios e instituciones públicas" (Bandia y Milton 2009: 1, nuestra traducción).

En nuestro estudio, tomamos pues en cuenta la labor de una gran diversidad de "agentes" mediadores: literatos políglotas, políticos o simples aficionados que contribuyeron a difundir las ideas positivistas. En muchos casos, aunque hay excepciones, se trataba de intelectuales de clases privilegiadas

y no siempre se indica el nombre del traductor. A menudo y al igual que el editor, o el prologuista, este aparece como autor secundario, de manera que tampoco es fácil saber si el "autor secundario" traduce, edita o prologa la obra. Del mismo modo, en la mayoría de los casos, se omite la lengua de origen a partir de la cual se ha hecho la traducción, lo cual dificulta la detección de traducciones indirectas. Asimismo, con frecuencia hemos encontrado registros duplicados, por lo cual ha sido igualmente difícil determinar si se trata de reediciones o reimpresiones de la misma obra. La prensa periódica, especialmente prolífica en el periodo que estudiamos, ha tenido que quedar fuera del estudio. Somos conscientes de la importancia de las revistas literarias y otras publicaciones culturales que sirvieron de vehículo, tanto para las traducciones como para los debates de los intelectuales de la época en torno al positivismo. Se trata, en efecto, de una tarea pendiente. A falta de poder hacerle justicia en este espacio mencionemos brevemente algunas de ellas. En Argentina, Alfredo Ferreira (1863-1938) y Pedro Scalabrini (18481916) fundan la revista mensual La Escuela Positiva, la cual circula entre 1895 y 1898. El Comité Positivista Argentino (creado en 1924), tendrá asimismo un órgano de expresión: la revista El Positivismo (1925), que tiene estrechas relaciones con la Revue Positiviste Internationale. En México, "entre 1878 y 1884, La Libertad se convierte en el órgano de los positivistas" (Beller, Méndez y Ramírez1973: 40). Publicada entre 1900 y 1914 , la Revista Positiva es otro de los vehículos de expresión de los "positivistas" mexicanos, en particular de Agustín Aragón (1870-1954). La revista publica muchas traducciones, no solo de filósofos positivistas. Finalmente, podemos mencionar la Revista Filosófica (1883-1884), desde la cual José Maria Vigil (1829-1909), criticó el positivismo. Los artículos ahí publicados, "salvo los de Vigil mismo- están traducidos del francés o del inglés" (Beller, Méndez y Ramírez 1973: 156). La revista retoma y se inspira de la publicación francesa Revue des Deux Mondes y del Dictionnaire des sciences philosophiques. 
que leían en otros idiomas y viajaban al extranjero. Muchos viajaron a París, asistieron a los cursos de Comte y entablaron relaciones con sus discípulos. Es el caso, por ejemplo, del chileno Jorge Lagarrigue (1854-1894) y de los mexicanos Pedro Contreras Elizalde (1820-1875) y Gabino Barreda (1818-1881) (González Navarro 1959: 119).

Pocos son los casos en que los actores hasta ahora considerados como los "precursores" o introductores del positivismo en Latinoamérica han sido también sus traductores. Antes bien, su papel parece haber sido mostrar la necesidad de tomar en cuenta estos textos e integrarlos a las discusiones que tenían lugar en sus respectivos países o para, inspirándose de esas lecturas, producir materiales que contribuyeran al "progreso" de las jóvenes naciones. Entre las excepciones más notables están Jorge Lagarrigue, quien firmó la traducción Principios de filosofía positiva de Comte (Santiago, Imprenta del Mercurio, 1875), y quien, junto con sus hermanos Luis y Juan Enrique, se involucró profundamente en la difusión del comtismo desde la Sociedad de la Ilustración.

Al tiempo que reconocemos la necesidad de incorporar a nuestro estudio la noción de "agentes de traducción", constatamos que el binomio texto de partida/texto de llegada limita, tal vez demasiado, el alcance de las investigaciones en historia de la traducción. Ésta nos ha enseñado que para el estudio de la importación de ideas y la construcción de literaturas, pueden considerarse otras prácticas de mediación lingüística: oral o escrita, íntegra o parcial, directa o indirecta. La traducción es un suelo propicio para la hibridación. Por medio de las traducciones, lo traducido se mezcla con lo que no lo es, los géneros literarios pierden sus contornos para dar lugar a otras formas de expresión, los autores devienen traductores y viceversa. Creemos que el papel de los editores forma parte del estudio de las "prácticas de mediación lingüística" y que en esa medida puede considerárseles como "agentes de traducción" con cuya intervención se renuevan repertorios culturales o "listas de opciones" estrechamente ligadas a la planificación cultural y a la construcción de "entidades colectivas" (Even-Zohar 2002: 45). Asimismo, su intervención articula "tres lógicas, la económica, la política y la cultural", las mismas que "rigen los intercambios culturales en proporciones y combinaciones que varían según el espacio y el tiempo" (Sapiro 2008: 21, nuestra traducción).

Antes de presentar la manera en que estas tres lógicas se articulan en la intervención de los "agentes de traducción" en el contexto que nos ocupa, conviene caracterizar la dinámica cultural y las lecturas que dan lugar a la importación de las ideas positivistas. Se trata de un ejercicio de contextualización 
que permitirá comprender mejor la naturaleza de los positivismos argentino y mexicano.

\subsection{Las lecturas "heterodoxas"}

A pesar de que recientemente Angenot reevaluó la tesis de las dos etapas del programa comtiano, para concluir que, en realidad, se trata de un sistema unitario en estrecha correspondencia con los "grandes relatos" del siglo XIX (2006: 17), la distinción entre las lecturas ortodoxa y heterodoxa será capital para los positivismos de Argentina y de México. El desarrollo de la propia obra de Comte contribuyó a que, tanto en Europa, como en Latinoamérica, se diera esta escisión (Subercaseaux 2004). La interpretación "heterodoxa" surge de una lectura selectiva que acepta las tesis expuestas en la primera etapa del programa comtiano, esto es la del Cours de philosophie positive (1830-1842), pero no la segunda, a saber, la del Catéchisme positiviste ou Sommaire exposition de la religión universelle (1852). En efecto, la "religión de la humanidad" hizo retroceder a muchos de los que en un principio recibieron la propuesta positivista con entusiasmo, entre ellos Émile Littré, discípulo de Comte, y John Stuart Mill, su interlocutor inglés.

Los positivismos de Argentina y de México se sitúan más bien del lado de las lecturas "heterodoxas". En ambos países, los representantes del positivismo conservan solo la propuesta del primer Comte y dejan de lado la "religión de la humanidad," combinando el positivismo con el liberalismo y con un evolucionismo inspirado en Spencer y en Darwin.

Por su obra Materialismo, Darwinismo, Positivismo. Diferencias y Semejanzas (1888), Pedro Scalabrini (1848-1916) es considerado como el "verdadero introductor del comtismo en Argentina" (Dozo 1971: 167). Es importante señalar, no obstante, que esta introducción se vio favorecida por las lecturas naturalistas que circulaban ya entre los intelectuales argentinos de las generaciones anteriores. Civilización y barbarie. Vida de Juan Facundo Quiroga. Aspecto físico, costumbres y hábitos de la República de Argentina (1845) de Domingo Faustino Sarmiento (1811-1888) y El génesis de nuestra raza (1862) de José Manuel Estrada son asimismo obras que prepararon el camino a la integración del primer Comte al positivismo argentino. En otros términos, antes de interesarse por el proyecto político propuesto por Comte, los positivistas argentinos se interesaron por el desarrollo de las ciencias. Si la década de 1880 es considerada como el acmé del positivismo en Argentina, debemos reconocer que antes que a Comte, se recurrió a los escritos de Darwin y de Spencer. Así, en 1877, año de la publicación de El Origen de las especies por medio de la selección natural o la conservación de las razas favorecidas en la lucha por 
la existencia (traducción de Enrique Godínez, publicada en Madrid por la editorial de José del Perojo), la Sociedad Científica Argentina hará de Darwin su tercer socio honorario. Un mes después de su muerte, en 1882, el Círculo Médico Argentino dedicará un homenaje al naturalista inglés, durante el cual el propio Sarmiento dará un discurso (Jalif de Bertranou 2009).

En suma, los positivistas argentinos encontraron en Comte y en Spencer la posibilidad de llevar al terreno social y político las ideas evolucionistas de Darwin. Los tres estados de Comte y el organicismo spenceriano nutrieron una concepción de la historia cuyo motor fue el progreso. Sarmiento, por ejemplo, dirá "con Spencer me entiendo bien porque andamos por el mismo camino" (Jalif de Bertranou 2009: 77). "El mismo camino" fue ante todo y en primer lugar el de la educación. Así, los positivistas argentinos justificaron la adopción del positivismo por la necesidad de lograr "la emancipación mental" (Zea 1980), cuyo primer paso fue la fundación de la Universidad de Buenos Aires en 1821. Este acontecimiento histórico marca el comienzo de un proceso de secularización influido en buena medida por la Ilustración francesa.

Este será uno de los puntos de contacto entre el positivismo mexicano y el argentino. De acuerdo con Beller, Méndez y Ramírez, "ya desde el siglo XVIII se empiezan a troquelar las bases materiales de la educación laica, libre de la influencia religiosa y la fundación de escuelas para la instrucción técnico-científica" (1973: 34). Los mismos autores coinciden en señalar que las posturas cientistas adoptadas por los positivistas mexicanos también tienen su primera inspiración en la Ilustración francesa (Beller, Méndez y Ramírez 1973: 121).

$\mathrm{Al}$ igual que en Argentina, en México los positivistas fueron liberales y desconfiaron de las lecturas ortodoxas. De ahí que "considerado como concepción filosófica, el positivismo no ha sido más que una posición que en realidad amalgamó bajo su nombre distintas tendencias, incluso contradictorias" (Beller, Méndez y Ramírez 1973: 103). Si puede considerarse a Gabino Barreda como el "precursor" de un positivismo que, aunque muy cercano de la propuesta comtiana, nunca fue "ortodoxo", también debe reconocerse que sus discípulos fueron poco a poco alejándose del comtismo para integrar las ideas de John Stuart Mill y de Herbert Spencer. La sustitución del lema comtiano "Orden, progreso y amor" por "Orden, progreso y libertad", propuesta por Gabino Barreda, es un hecho bien conocido en la historiografía del positivismo en México y constituye un ejemplo paradigmático de las negociaciones que los positivistas tuvieron que llevar a cabo para introducir el ideario comtiano en México (Zea 1980). 
Entre la muerte de Comte (1857) y la creación de la Escuela Nacional Preparatoria (1867), "aparecieron obras trascendentales: El origen de las especies, de Darwin; Introducción a la medicina experimental, de Claude Bernard; El utilitarismo, de Mill, y los primeros tomos del Sistema, de Herbert Spencer" (Beller, Méndez y Ramírez 1973: 127). Aunque las traducciones al español de estas obras habrán aún de esperar algunos años, su circulación en lengua extranjera permitirá que, desde fines del siglo XIX, el panorama de las ideas positivistas en México se oriente hacia la "corriente spenceriana" y el utilitarismo de Stuart Mill (Beller, Méndez y Ramírez 1973: 45). Así, cuando a principios del siglo XX, Horacio Barreda defienda el positivismo introducido en México por su padre, no lo hará en nombre del sistema comtiano, sino apelando al método "ecléctico" de razonamiento útil para plantear y resolver problemas. La fundación de la Asociación Metodófila el 4 de febrero de 1877 sanciona esta reducción del sistema positivista asimilado ahora al "método científico" (Beller, Méndez y Ramírez 1973: 129).

\section{Editores y traductores}

La circulación de impresos en Hispanoamérica en el siglo XIX está íntimamente ligada al mercado editorial de los antiguos centros coloniales, y a la vez está sujeta a la batalla que editores de ambos lados del Atlántico libran por el mercado lector hispanohablante. A decir de Pura Fernández, ya en la década de 1850, más de la cuarta parte de los libros editados en Francia se exportaban a Latinoamérica (1998: 174). Libreros, impresores, escritores e intelectuales desempeñan un papel central en dicho mercado editorial que no es independiente de movimientos literarios y de planificación cultural. Asimismo, de acuerdo con dicha autora:

La mejora y el abaratamiento de los medios de transporte y de los servicios postales, la concentración urbana, así como los planes de alfabetización abordados por los gobiernos y la reducción de los costes de producción, fomentaron las relaciones comerciales y políticas entre Europa y América y, con ellas, la internacionalización del mercado editorial, favorecido por la creciente permisividad de la normativa legal de imprenta (Fernández 1998: 166).

En particular, la experiencia de Francia y de España en el comercio librero internacional les permite librar la batalla por el mercado editorial de las nuevas repúblicas latinoamericanas y, en la segunda mitad del siglo XIX, libreros y editores franceses se lanzan a la conquista del mercado latinoamericano. Los hermanos Garnier, por ejemplo, lograrán monopolizar el libro escolar en las nuevas repúblicas por un breve periodo de tiempo (Fernández 1998). 
Al margen de un mercado latinoamericano cada vez más importante, el cual se encontraba en manos de editores como Garnier pero también Bouret, Baudry, Colin y otros, a fines del siglo XIX y a principios del XX, los editores españoles reaccionan contra lo que consideran una política de colonización cultural caracterizada, según algunos observadores, por el mal gusto y la mala calidad. Así lo escribe Juan Valera en una carta a Menéndez Pelayo:

Una publicación de libros castellanos para América, hecha en grande escala y con sentido común [...] contribuiría al esplendor y difusión de nuestra literatura y anularía y sepultaría para siempre en el olvido las malas y groseras ediciones que salen de las prensas de París, atestadas con feísimas e intolerables erratas e hijas de un sórdido anhelo de lucro, incompatible con todo buen gusto literario y tipográfico (citado por Fernández 1998: 182).

De hecho, como lo indica Larraz Elorriaga, a fines del siglo XIX, los editores españoles se servían de un discurso que enarbolaba la lengua española como estandarte de una lucha contra "la proliferación de libros alemanes y franceses en lengua española por toda América Latina" (2011: 131), la cual era percibida como "un desdoro para España e incluso una desnaturalización cultural de las antiguas colonias" (2011: 131)

Además, como lo señala Gutiérrez Rodilla, tras el letargo causado por la censura inquisitorial de los siglos XVI y XVII, la traducción científica al español experimenta un auge en los siglos XVIII y XIX, sobre todo desde el francés, lengua intermediaria desde la cual llegaron al español obras del "alemán, italiano, portugués, griego o latín" debido a "la mayor familiaridad de los traductores con la lengua gala" (2009: 236). De alguna manera, las traducciones positivistas que circulan a fines del siglo XIX dan continuidad a este movimiento de traducciones científicas.

Debido a que, en el periodo que nos ocupa, los editores y libreros europeos e hispanoamericanos desarrollan sus actividades paralelamente, conviene tratarlos de manera independiente. A continuación dedicamos una sección a los libros europeos distribuidos en Hispanoamérica y otra a la actividad del mercado editorial "local".

\subsection{Los libros europeos en Hispanoamérica}

Los catálogos bibliográficos consultados dan cuenta de la presencia de empresas editoriales francesas y españolas cuyos tirajes y publicaciones corresponden a proyectos de divulgación, dirigidos a un público más amplio, así como también de editores e intelectuales comprometidos con movimientos culturales cuyo lectorado es más restringido. Garnier Frères y Espasa Calpe, por ejemplo, exportan a las nuevas repúblicas latinoamericanas títulos 
originales y traducciones al español de todo tipo de obras. Sin ser los únicos libreros-editores en trascender el mercado europeo, sus numerosas colecciones (Biblioteca selecta para niños, Biblioteca selecta para la juventud, Biblioteca de los novelistas, Biblioteca de Autores célebres, etc. ) toman ventaja de las cambiantes normativas de imprenta de un país a otro e inundan el mercado librero latinoamericano.

Conviene aquí hacer hincapié en el proyecto colectivo del Diccionario Enciclopédico de la lengua castellana de Garnier. La obra está destinada al mercado latinoamericano y se publica en dos volúmenes en 1895, reuniendo las contribuciones de un gran número de colaboradores hispanohablantes establecidos en la capital francesa (Fernández 1999), quienes escriben, adaptan y traducen una gran cantidad de material escrito destinado a ser incorporado en esta enciclopedia. Uno de ellos fue Nicolás Estévanez Murphy (18381914). Militar federalista y republicano, nombrado gobernador de Madrid al proclamarse la República en 1873, ex ministro de Pi y Margall, Estévanez se exilia sucesivamente a Portugal, Cuba, Estados Unidos, México y París tras la restauración del régimen monárquico. Su trayectoria como autor y traductor está estrechamente relacionada con Garnier, para la cual tradujo durante unos treinta años de manera constante, como parte del grupo de letrados vinculados al Diccionario. Muchas de sus traducciones filosóficas son tardías o se publicarán de manera póstuma, como el Catecismo positivista. Exposición sumaria de la religión universal, de Comte (Garnier 1913 y 1920). Tradujo una fascinante miscelánea de asuntos militares, filosofía, geografía, historia y literatura para niños. En filosofía, además de Comte, traduce a Séneca (Obras escogidas, 1914), Cicerón (Obras escogidas, 1914), Aristóteles (La Política, 1920) y Montesquieu (Del espiritu de las letras, 1921), para la misma editorial.

Charles Bouret, otro editor francés con vocación internacional, comparte con Garnier la distribución a gran escala de libros y manuales en español. Comienza sus actividades editoriales alrededor de 1820 en asociación con Jules Rosa (Librería de Rosa y Bouret). A la muerte de Bouret, la editorial adopta el nombre "Viuda de Bouret" (Fernández 1998: 175-176), posteriormente establecida en la ciudad de México. Esta editorial francesa dominó el comercio del libro en ese país durante muchos años, posiblemente, como lo indica Fernández (1998: 174) favorecida en sus gestiones por la intervención francesa de 1862 y sus consecuencias. Con su sello aparece en 1897 en París (reimpreso en México en 1925) el Resumen sintético del sistema de lógica de John Stuart Mill, firmado por y con notas complementarias de Ezequiel Adeodato Chávez Lavista (1868-1946). También publica el Resumen sintético de los principios de moral de Herbert Spencer (París, 1898), obra que será reimpresa 
en México en tres ocasiones (1905, 1910 y 1922). No debe pasarse por alto que el autor de esta traducción-comentario, es considerado, junto con Gabino Barreda y Justo Sierra, como uno de los intelectuales que más contribuyó a la difusión del positivismo en México (Díaz Zermeño 1999). No obstante, su labor como comentarista dista de servir de "introducción" a las obras de Mill y de Spencer en el mundo hispanohablante. Lo precede en la tarea de difundir las ideas positivistas en español, Eduardo Zamacois y Quintana (1876-1971), ${ }^{3}$ cuya traducción de La clasificación de las ciencias de Spencer se publica en 1889 en la Biblioteca Económica Filosófica fundada por Antonio Zozaya y Jou (1859-1943).

En España, la publicación y difusión del positivismo es un tanto tardía, debido a la influencia del krausismo. De acuerdo con Soriano Nieto (2009: 252), "hasta la década de 1870 no se introdujo, a través de José del Perojo y sus contemporáneos, un interés por la filosofía positivista".

A diferencia de Garnier y de Bouret, cuya orientación es evidentemente comercial, las traducciones de José del Perojo y los proyectos editoriales como el de la Biblioteca Económica Filosófica de Zozaya (Lafarga y Pegenaute 2009: 1190-1191), La España Moderna (1889-1914) y Revista de Occidente se destinan a un público culto. Ocupémonos primero de la "Biblioteca" de Zozaya. Filósofo, escritor y traductor de un gran número de obras de filosofía, política, ciencia y moral y de convicción republicana, como muchos republicanos españoles debe exiliarse a México a la llegada de Franco al poder. En su "Biblioteca" aparecieron sus propias traducciones del Catecismo positivista ó Sumaria exposición de la religión universal en trece diálogos sistemáticos entre una mujer y un sacerdote de la Humanidad, de Comte, publicado en 1886 y luego en 1889 (Imprenta de Manuel Minuesa), y de El utilitarismo, de Stuart Mill (Dirección y Administración, 1891).

También destinada a un público de especialistas, La España Moderna (1889-1914) es creada por José Lázaro Galdeano (1862-1947) en aras de la restauración del "depauperado mundo cultural de la España contemporánea" (Asún, 1981-82: 140). Entre los traductores relacionados con esta editorial, se encontraban algunos de los intelectuales krausistas más respetados de la época, ya que "Lázaro intentó buscar aquellas figuras más capaces de comprender el texto original cuando éste manejaba un código científico" (Asún 1981-82: 152). Uno de ellos fue Miguel de Unamuno (1864-1936), protégé de

3. Zamacois nació en Cuba de padres españoles, estudió en París y en Madrid y murió en Argentina. Escribió novelas realistas, naturalistas y eróticas, y estuvo relacionado con la generación del 98 (cf. Archivo Histórico Nacional). 
Galdeano, quien firmó diecisiete traducciones de Spencer, autor que también tradujeron para la misma editorial Adolfo Posada (1860-1944) y Leopoldo Palacios Morini (1876-1952). Así, De las leyes generales, La moral, El organismo social, El progreso. Su ley y su causa y Ética de las prisiones de Spencer serán publicadas en 1895. De Darwin, La España Moderna publicó El viaje de un naturalista alrededor del mundo en 1899, en la traducción de Luis Domènech. En esta casa editorial aparecen igualmente en 1905 los Principios de filosofía positiva de Comte que corresponden a las dos primeras lecciones del Cours. Sobre esta última obra, en su estudio histórico sobre La España Moderna, Raquel Asún indica que "existe una traducción anterior de 1886-87, realizada por la Biblioteca filosófica [sic. ]. La de La España Moderna intentó un mayor rigor terminológico" (1981-82: 164). Podría ser que la traducción a la que la autora se refiere se haya publicado en la colección creada por Zozaya, la Biblioteca Económica Filosófica. Asimismo, de John Stuart Mill La España Moderna publicó los Estudios sobre la religión, traducido por Luis Terán (1906). Hacia 1895, se registra un descenso en la producción y a partir de 1896 La España Moderna "se fue convirtiendo en una editorial científica", cuyas publicaciones tenían un tono muy elitista (Asún 1981-82: 147-48).

La difusión de textos filosóficos llevada a cabo por la Revista de Occidente, fundada por José Ortega y Gasset (1883-1955) en 1923, primero como una publicación periódica y luego como casa editorial, es importantísima. En lo que toca a las traducciones del positivismo por la editorial Revista de Occidente, es necesario mencionar el Discurso sobre el espiritu positivo: orden y progreso de Comte, traducido por Julián Marías (1914-2005) en Madrid en 1934.

Lo anterior no debería llevarnos a la conclusión de que España no tuvo empresas de edición y distribución a gran escala como las francesas Garnier y Bouret. Una de ellas fue Espasa, (hoy en día, parte del grupo editorial Planeta), fundada en Barcelona por José Espasa Anguera, durante la segunda mitad del siglo XIX. Aunque no se limitaba a un público lector de especialistas, todo parece indicar que reclutaba a sus traductores entre los escritores e intelectuales de la época. En lo que toca a las traducciones de obras positivistas, Antonio de Zulueta y Escolano (1885-1871) publicó allí su versión de El origen de las especies (1921), hecha a partir de la sexta edición del texto de Darwin y considerada como la mejor traducción al español de esa obra. Zulueta "fue uno de los pioneros de los estudios de genética en España y conservador del Museo de Ciencias Naturales de Madrid" (Santoyo 2009: 286).

En 1926, Espasa se funde con Calpe (sello de La Papelera Española) y establece delegaciones en Latinoamérica, particularmente en Buenos Aires. Dan constancia de la vitalidad y auge cultural de dicha empresa, los fascículos de 
la Enciclopedia Universal Ilustrada Europe-Americana, también conocida como Enciclopedia Espasa, publicada a partir de 1908. Después de esta fecha y hasta 1930, aparecen 72 volúmenes. De ahí que Espasa Calpe comparta con Garnier, en cuanto a la producción cultural de la época, el estatus de casa editorial de gran producción que vende y exporta grandes cantidades de libros de manera indiscriminada.

Sin embargo, este no parecía ser el proyecto inicial. En 1921, Calpe (Madrid) publica la Autobiografía de John Stuart Mill, en la traducción de Juan Uña Gómez (1838-1909). La obra es reeditada por Espasa Calpe Argentina en 1939 y 1945, y parece más bien estar destinada a un público especializado. Su traductor, Juan Uña, es licenciado en Filosofía y Letras y egresado de la Escuela de Diplomática. Pedagogo y adepto del krausismo es, durante la Primera República española (1868-1874), director de la Enseñanza Pública y diputado en Las Cortes. Funda la Institución Libre de Enseñanza y dirige la revista La Enseñanza. Progresista y socialmente comprometido, funda dos escuelas para niños desfavorecidos. Su obra de traductor incluye también una versión española de la Metafísica de Kant.

Otro proyecto editorial español de largo alcance es el emprendido por Francisco Sempere y la editorial homónima (posteriormente Prometeo). Dicha editorial publicó algunos trabajos de Spencer en español, como El individuo contra el Estado (traducción de Gómez Pinilla, 1884), Las ceremonias de la vida (traducción de G. Sánchez, 1911) y El progreso (traducción de Eugenio López, 1911). De Darwin, publicó La expresión de las emociones en los hombres y en los animales (traducción de Eusebio Heras, s. f. ), una versión de El origen de las especies (traducción de A. López White, 1903) y otra de Mi viaje alrededor del mundo (traducción de Constantino Piquer, s. f. ). Aunque eran "bastante descuidadas", las traducciones de Sempere tenían el objetivo de divulgar las ideas revolucionarias de la Europa de su época. De acuerdo con una carta del librero a Unamuno, sus libros circulaban también en América (Pérez de la Dehesa, 1969), en particular, en Argentina, donde, a través de su colección Biblioteca Blanca, "militantes políticos y obreros ilustrados" tenían acceso a "las más importantes obras de la literatura universal, de las vanguardias y del pensamiento social" pues los volúmenes solo costaban 30 centavos (Romero1990: 45).

Se inscribe en la misma tendencia de difusión y vulgarización el proyecto editorial de Juan Bautista Bergua (1892-1991), quien heredó el oficio librero de su padre, Juan Bergua López, y quien fundó en 1927 la Librería-Editorial Bergua (hoy en día Ediciones Ibéricas). De particular relevancia para nuestro estudio es su colección Biblioteca de Bolsillo, "libros para llevar en el 
bolsillo literalmente y leer en cualquier rato libre y también, para poder formar sin grandes gastos una biblioteca culta" ("Nuestro origen", Ediciones Ibéricas, 2007). La pasión librera y el gusto por los idiomas se conjugaron con el contexto de la República española y dieron al proyecto editorial de Juan Bautista Bergua mucho éxito en los años de la República. Además del trabajo de edición, Bergua tradujo unos cincuenta títulos clásicos (el Corán, la Iliada y la Odisea), así como también las obras completas de Platón anotadas y acompañadas de textos introductorios. Entre los textos evolucionistas que puso en circulación en su colección de bolsillo, deben mencionarse los dos tomos de El origen del hombre y la selección en relación al sexo, traducidos por M. J. Barroso-Bonzón en 1933. Como lo indica el traductor al final del prólogo donde explica la importancia de la obra y lamenta la ignorancia española acerca de Darwin:

Nos sentimos orgullosos de dar la primera edición completa en nuestro idioma, adicionada con partes, desconocidas hasta ahora en nuestro país, porque sólo las incluyen las ediciones inglesas; y muy orgullosos así mismo de no omitir nada ni en texto ni grabados, no obstante el carácter económico, de edición de bolsillo, que ostenta la Biblioteca a que este ORIGEN DEL HOMBRE pertenece (Barroso-Bonzón, en su traducción de Darwin 1966: 14).

Es momento ahora de volver la mirada hacia el panorama librero y editorial latinoamericano, en particular el de Argentina y de México. A pesar de la insoslayable presencia de los libros europeos en Hispanoamérica, la producción y circulación de impresos "locales" experimenta cambios importantes. Si bien la fundación de la Real Imprenta de los Niños Expósitos data de 1780 y la llegada de la imprenta a México de 1539 (poco después de la fundación de la Real y Pontificia Universidad), solo en el siglo XIX podemos observar el desarrollo de una industria editorial "local". Los libreros primero y, luego, los editores, contribuyen a la difusión de impresos, ya sea bajo la forma de publicaciones periódicas, esto es, revistas y folletos, o de libros para hacer frente a la demanda que las editoriales europeas no logran satisfacer.

\subsection{Los libros americanos: Argentina y México}

En el periodo comprendido entre 1850 y 1950, la producción y circulación de impresos experimenta cambios importantes. Especialmente en la primera mitad del siglo XX, ya sea bajo la forma de publicaciones periódicas, esto es, revistas y folletos, o de libros, se observa el desarrollo de una industria editorial "local" constituida por libreros e impresores que poco a poco se transforman en editores. 
En Argentina, en particular, el periodo de entreguerras ve surgir "ambiciosos proyectos editoriales" que echan mano de los esfuerzos alfabetizadores llevados a cabo desde principios de siglo (Romero1990: 46). Dichos esfuerzos resultan en el incremento de un lectorado al cual editores y libreros buscan cautivar. Si bien esta "ampliación del público lector coincidía con la definición de un mundo intelectual especializado, de escritores profesionales, cenáculos, revistas y formas propias de consagración" (Romero 1990: 46), también se hace necesario satisfacer la demanda de textos literarios y de divulgación cuyo objeto era más bien contribuir a "poner ciertas obras al alcance del pueblo" (Romero 1990: 47). Las traducciones positivistas corresponden a esta segunda intención. Publicadas en su mayoría en el siglo XX, compartían con las traducciones literarias la siguiente característica: "no atendían tanto a la incorporación de lo nuevo como a la democratización del consumo de libros y la ampliación del público lector" (Willson 2004: 47).

Así, Pedro García, inmigrante proveniente de una familia de libreros, fundó La librería El Ateneo en 1912. Su catálogo, uno de los más influyentes en ese país, estaba constituido por manuales, obras de literatura, ciencia, derecho, bellas artes y literatura infantil. En la publicidad distribuida en 1928 por el diario argentino La Nación se decía: "Cuantos necesitan un libro, ya se trate de una obra científica o literaria, tienen la certidumbre de encontrarlo al menor precio en nuestra casa. Hacemos créditos y vendemos al interior: pídanos catálogo de las materias que le interesen".

Particularmente exitosa fue la colección Clásicos Inolvidables, en la que se tradujeron textos filosóficos (Platón, Sófocles) y literatura (Shakespeare). Durante la Guerra Civil española y el ascenso del franquismo al poder acudieron a ella muchos editores que no podían publicar sus libros en España y la frecuentaron también autores como Jorge Luis Borges, Adolfo Bioy Casares, Victoria Ocampo y Leopoldo Marechal, entre otros.

En lo que respecta a la publicación de las obras que nos interesan, en 1942, publica el Viaje de un naturalista alrededor del mundo de Darwin, en la traducción de J. Hubert. La portada de esta traducción indica que se trata de una Edición completa. Ampliada con más de 120 ilustraciones de la época. Seleccionadas y ordenadas por Joaquín Gil. Joaquín Gil Guiñón empezó a publicar en la década de 1930 en Barcelona y dejó su editorial en manos de un hermano para emigrar a Buenos Aires antes de la Guerra Civil. Los títulos de "su" colección Obras Maestras (traducciones españolas de grandes clásicos) empiezan a circular en Barcelona en 1944, bajo el doble sello Joaquín Gil-Editorial Iberia. El Viaje de Darwin se abre con una larga cita del autor, seguida por un índice de grabados, un índice y un texto de Joaquín Gil titulado Presentación de la 
edición argentina. La insistencia por mostrar que se trata de una edición especialmente adaptada para Argentina se comprende mejor si se menciona que la misma obra de Darwin se publicó en Madrid con anterioridad (1933). Por ello el editor indica que "se han incluido en esta edición argentina antiguos grabados que nos muestran costumbres de Buenos Aires en el primer tercio del siglo pasado" (Gil, en Darwin 1966: 19). La presentación prosigue en los siguientes términos:

Confiamos en que esta edición, la primera en lengua castellana, que se presenta ilustrada con tal profusión de interesantes y valiosos grabados, recibirá por parte de los lectores argentinos y continentales de habla castellana, una favorable acogida. Con ello consideraremos que ha sido lo suficiente acertada nuestra selección de grabados para ilustrarla y la presentación de conjunto dada a la obra tal como ahora aparece (Gil, en Darwin 1966: 19).

Spencer será profusamente traducido y editado en Argentina, pues como vimos anteriormente, las lecturas positivistas en ese país siguieron una tendencia heterodoxa que combinó las aportaciones del primer Comte, Darwin y Spencer. De este último, aparece Clasificación de las ciencias, en la editorial Anaconda (Buenos Aires, s. f. ), la cual tomó el lugar de la antigua editorial Minerva, fundada por Santiago y Leonardo Glusberg en 1924 (Gasió 2008: 48). Es muy probable que la Clasificación de las ciencias que publicó Anaconda sea una reimpresión de la versión de Eduardo Zamacois y Quintana (Madrid, 1889) para la Biblioteca Económica Filosófica de Zozaya.

Entre los proyectos de divulgación más importantes de la Argentina en el periodo de entreguerras debemos igualmente mencionar a la editorial Tor, fundada en 1916 por Juan Carlos Torrendell (1895-1961), editorial que "hasta su cierre en 1971 produjo alrededor de 10000 títulos de libros y 2000 de revistas de diversos géneros" (Abraham 2012: 14) Hasta los años treinta publicó ensayos y autores entonces poco conocidos, como Jorge Luis Borges. Tor propuso libros y fascículos que permitían leer a buen precio y de manera regular. En una segunda época de la editorial, esta amplió la gama de los géneros que publicaba y se dedicó a producir ediciones baratas de clásicos, ciencia ficción y otros géneros a precios accesibles. De especial interés para lo que aquí nos concierne fue su Nueva Biblioteca Filosófica, que ofreció al público obras de autores de renombre a bajo precio. En esta categoría entra El Espiritu Positivo (s. f. ), un fascículo de formato pequeño cuya portada (fondo de color naranja con el clásico retrato de Comte) indica en una pequeña etiqueta redonda el precio (30 centavos) y la mención Aparece los miércoles y es de TOR. Creación y Evolución de Spencer apareció en esta colección, con el mismo formato y presentación en 1941. 
La difusión de los textos positivistas en México es un tanto diferente de la que podemos observar en Argentina. La diferencia principal consiste en que, mientras en Argentina la labor de edición y difusión de las obras positivistas quedó en manos de editores y libreros independientes, en México, debido a la importancia que el positivismo tuvo para la restructuración del sistema educativo y para la definición de la ideología oficial, con mucha frecuencia, estas obras se difundieron a costa o por encargo de instituciones gubernamentales. Este es, al menos el caso, de Los Antiguos Mexicanos (1896) y El Antiguo Yucatán (1898) de Herbert Spencer, traducidas por los hermanos Daniel y Genaro García (1867-1920) y publicadas por la Oficina Tipográfica de la Secretaría de Fomento (actualmente Secretaría de Economía). Ambas obras fueron extraídas de Descriptive Sociology, una serie de diecisiete volúmenes de datos etnográficos e históricos a cargo de Spencer. El Antiguo Yucatán, en particular, toma de esa obra la parte relativa a la península de Yucatán. En el prólogo, los traductores dedican esta selección a Porfirio Díaz y añaden una nota que vale la pena citar in-extenso:

En el prólogo de nuestra traducción de Los Antiguos Mexicanos advertimos que en la obra inglesa existen mal traducidos, y en ocasiones adulterados, muchos pasajes de nuestros antiguos historiadores. Otro tanto debemos decir aquí de El Antiguo Yucatán, agregando que si en aquella traducción respetamos hasta donde nos fue posible la forma que tales pasajes ofrecen en la obra inglesa y nos limitamos a fijar su sentido verdadero en vista de los textos originales, hoy en la presente traducción hemos preferido transcribir literalmente estos últimos tomándolos de autorizadas ediciones con el objeto de evitar toda inexactitud en nuestro trabajo. (Daniel y Genaro García 1898: 7)

En otros términos, Daniel y Genaro García se dan a la tarea de corregir los errores que encuentran en la obra de Spencer y que atribuyen al hecho de que el autor no conoce el español, como "lo manifiesta el propio Spencer en una carta dirigida a uno de nosotros" (Daniel y Genaro García 1898: 7). Los traductores entran, pues, en contacto con el autor y, podemos suponer, obtienen de él la autorización de hacer la selección y modificaciones a Descriptive Sociology para publicarla como El Antiguo Yucatán. Reconocido bibliófilo y estudioso de la historia mexicana, Genaro García añade a su traducción un anexo que da cuenta de su profundo conocimiento de las fuentes historiográficas necesarias para "corregir" las deficiencias de la versión inglesa. Entre las numerosas obras listadas en ese anexo se cuentan La historia antigua de México de Clavijero, las Cartas de Relación de Cortés y la Relación de las cosas de Yucatán de Fray Diego de Landa. Las traducciones de Spencer así publicadas son representativas de un proceso de reapropiación justificada por un discurso nacionalista. 
La traducción Los más curiosos animales de América (1944) de Darwin es quizá un segundo ejemplo de este fenómeno. Publicada en la colección "Cuadernos de Cultura" quedan pocos rastros de la imprenta donde vio la luz y de la historia de su publicación. Podemos, sin embargo, suponer que se trata igualmente de una selección de Mi viaje alrededor del mundo (1900 ca.) de Darwin, con fines de divulgación.

Finalmente, debemos mencionar los Primeros ensayos atribuidos a Comte, traducidos por Francisco Giner de los Ríos (1839-1915) y publicados póstumamente por el Fondo de Cultura Económica (1942). En la misma casa editorial, aparecerán los Principios de economía política, con algunas de sus aplicaciones a la filosofía social de John Stuart Mill, traducidos por Teodoro Ortíz en 1943 (reimpresión en 1951). Creada en 1934 por Daniel Cosío Villegas (1898-1976), esta casa editorial surgió justamente para satisfacer la necesidad de traducciones en ciencias sociales para las facultades universitarias recién creadas en la Universidad Nacional Autónoma de México. A fines de los años treinta, un grupo de intelectuales españoles, entre ellos Francisco Giner de los Ríos, mencionado anteriormente, se integra a las actividades editoriales y de traducción del Fondo y contribuye de manera importantísima a la consolidación de sus colecciones (sociología, economía, breviarios, biblioteca mexicana, etc. ) (Díaz Arciniega 1996). Puesto que uno de los objetivos de la editorial era la publicación de traducciones, no debemos sorprendernos de que haya sido, precisamente, en el seno de sus actividades editoriales que la actividad del traductor comenzó a profesionalizarse en México. De manera que, en esta empresa editorial, se combinó un proyecto de planificación cultural estrechamente vinculado a la educación universitaria, con el rigor exigido a la traducción especializada.

Hemos de reconocer que este recuento bibliográfico, muy especialmente en lo que concierne a las obras de Darwin en español, es dolorosamente fragmentario. Debemos, pues, contentarnos con estas referencias que en modo alguno agotan la información existente sobre la obra traducida del naturalista. Esto se debe sin lugar a dudas al hecho de que es "muy probable que ninguna obra de carácter científico, desde luego, no del siglo XIX, haya alcanzado en España difusión semejante a la de El origen de las especies" (Santoyo 2009: 286). Señalemos que el lector interesado puede acudir a la Bibliografía Crítica Ilustrada de las Obras de Darwin en España (1857-2008) (Gómez Blanco y Josa Llorca 2009), publicada con motivo del bicentenario del nacimiento de Charles Darwin. 


\section{Conclusiones}

En estas páginas hemos querido estudiar la circulación de las ideas positivistas en Argentina y México en el periodo comprendido entre 1850 y 1950. La delimitación del contexto espacio-temporal de nuestro estudio ha dejado sin duda zonas inexploradas, cuyo análisis no solo es deseable, sino necesario. La aportación de este trabajo bibliográfico para la vertiente histórica de los estudios de traducción, creemos, consiste, en primer lugar, en considerar las prácticas editoriales como objeto de estudio de la disciplina. El papel desempeñado por los editores hispanoamericanos de los que nos hemos ocupado en estas páginas permite constatar que para la circulación de las ideas positivistas no solo cuentan los traductores comprometidos, sino su inserción en procesos más complejos que dan lugar a la producción de repertorios culturales. Esto no significa que su labor traductora no sea importante. El matiz que buscamos mostrar es que esta actividad traductora no necesariamente responde a una militancia política o intelectual. Al considerar a intelectuales, editores y traductores como "agentes de traducción" hemos querido recobrar la complejidad de la circulación de las ideas positivistas y mirar nuestro objeto de estudio no solo desde la perspectiva de los "agentes" involucrados, sino también desde aquella del mercado editorial internacional. Proceder de esta manera es pues una invitación abierta a repensar la tesis según la cual los traductores del positivismo fueron igualmente sus precursores e introductores. Como hemos podido observar, participan igualmente instituciones culturales, casas editoriales e incluso mecenas que buscan contribuir a la producción cultural en general o proteger a algún escritor que traduce para procurarse un ingreso. Sin renunciar a la importancia de las trayectorias sociales de los traductores y editores estudiados, nuestro propósito ha sido también mostrar lo que un panorama meramente biográfico podría ocultar, esto es, los factores explicativos que revelan el entramado de circunstancias socioculturales, relaciones, conflictos y negociaciones e incluso azares o contingencias que conforman cualquier empresa de traducción en el sentido sociológico del término.

En segundo lugar, hemos juzgado necesario ampliar el panorama de estudio de esta manera porque, con frecuencia, al relacionar de manera unívoca el positivismo latinoamericano con el positivismo europeo, la complejidad de los positivismos latinoamericanos puede pasar desapercibida. Así, seguimos a Woodward cuando afirma que: "ideological currents are never as exact in practice, as they may appear to the scholar in theory, nor is their influence confined to direct, measurable, factors" (Woodward 1971: x). En otros términos, al mostrar que los positivismos argentino y mexicano se constituyeron con lecturas selectivas y combinando las obras de Comte, Darwin, Spencer 
y Mill, quisimos resaltar la falta de correspondencia entre el "original" y su "traducción" e insistir en la necesidad de adoptar perspectivas teóricas que permitan restablecer la complejidad de la circulación de las ideas.

La simultaneidad con que, en el contexto estudiado, circulaban originales $\mathrm{y}$ traducciones nos ha llevado igualmente a reconsiderar la tesis que equipara la traducción de una obra a su introducción en el contexto receptor. Como lo hemos visto en los casos argentino y mexicano, esto no siempre sucede de esta manera. En Argentina y en México, la circulación de obras positivistas, originales y traducidas, está determinada en gran medida por un interdiscurso que precede a las lecturas de aquellos que participan en la importación de esta corriente de pensamiento y, por supuesto, por el contexto político y cultural en el que se insertan. En suma, para constituirse, los positivismos de Argentina y de México no esperaron a que las ideas que tomaron por sustento circularan en español. La importación y la traducción de las filosofías amalgamadas bajo la etiqueta "positivista" ocurrieron con frecuencia de manera simultánea. Aun más, estos positivismos son per se el resultado de traducciones selectivas y de reescrituras producidas en la urgencia de la organización nacional. Las traducciones positivistas lato y stricto sensu, forman parte de proyectos de planificación cultural en los cuales participan políticos, intelectuales, libreros, impresores, escritores y traductores. La actividad editorial de la época no es en absoluto ajena a las empresas sociales y culturales que dan lugar a un repertorio bibliográfico muy diverso en el cual se insertan los textos positivistas.

\section{Bibliografía}

ABRAHAm, Carlos. (2012) La editorial TOR. Medio siglo de libros populares. Temperley: Tren en movimiento.

ANGENOT, Marc. (2006) "Tombeau d'Auguste Comte." Discours social 26.

ARChivo Histórico Nacional. "Zamacois y Quintana, Eduardo." Versión electrónica <http: //censoarchivos. mcu. es/CensoGuia/fondoDetail.htm? $\mathrm{id}=1095953>$.

ARDAO, Arturo. (1963) "Assimilation and Transformation of Positivism in Latin America." Journal of the History of Ideas 24: 4, pp. 515-522.

Asún EsCarTín, Raquel. (1981-1982) "La editorial 'La España Moderna'." Archivum. Revista de la Facultad de Filología 31-32, pp. 133-200.

BandiA, Paul \& John Milton (eds.) (2009) Agents of Translation. Amsterdam/ Philadelphia: John Benjamins.

Beller, Walter; Bernardo Méndez \& Santiago Ramírez. (1973) El positivismo mexicano. México: UAM-Xochimilco. 
DARWIN, Charles \& M. J. Barroso Bonzón (traductor). (1966) El origen del hombre y la selección en relación al sexo, t. 1, 2a ed. Madrid: Ediciones Ibéricas.

Díaz Arciniega, Victor. (1996) Historia de la casa. Fondo de Cultura Económica (1934-1996). México: FCE.

DíAz Zermeño, Héctor. (1999) "Ezequiel A. Chávez: Rasgos de su trayectoria y pensamiento político educativo". Perfiles Educativos 83-84, pp. 69-81.

Dozo, Luis Adolfo. (1971) "Alfredo Ferreira y el positivismo argentino". Cuyo. Anuario del Pensamiento Argentino 7, pp. 160-175.

Ediciones Ibéricas (2007) "Nuestro origen". Versión electrónica: <http: //www. edicionesibericas. es/nuestro_origen. php>.

EVEN-ZOHAR, Itamar (2002) "Culture Planning and Cultural Resistance in the Making and Maintaining of Entities." Sun Yat-sen Journal of Humanities 14, pp. 45-52.

FERNÁNDEZ, Pura. (1998) "El monopolio del mercado internacional de impresos en castellano en el siglo XIX: Francia, España y la 'ruta' de Hispanoamérica." Bulletin hispanique 100: 1, pp. 165-190.

FERnández, Pura. (1999) "La editorial Garnier de París y la difusión del patrimonio bibliográfico en castellano en el siglo XIX." En: Varios autores. (1999) Tesphiliestade dora: miscelánea léxica en memoria de Conchita Serrano. Madrid: Consejo Superior de Investigaciones Científicas, pp. 603-612.

GASIÓ, Guillermo. (2008) El más caro de los lujos: Primera Exposición Nacional del Libro, Teatro Cervantes, septiembre de 1928. Buenos Aires: Teseo.

GonZÁlez NaVArro, Moisés. (1959) "Los positivistas mexicanos en Francia". Historia mexicana 9: 1 (33) (jul. -sept.), pp. 119-129.

Gomis BlanCO, Alberto \& Jaume Josa Llorca. (2009) Bibliografía Crítica Ilustrada de las Obras de Darwin en España (1857-2008). Madrid: Consejo Superior de Investigaciones Científicas.

GutiÉRrez Rodilla, Bertha. (2009) "Científica, traducción". En: Lafarga, Francisco \& Luis Pegenaute (eds.) 2009. Diccionario histórico de la traducción en España. Madrid: Gredos, pp. 230-239.

JALIF DE Bertranou, Clara Alicia. (2009) "Charles Darwin: un navegante de los tiempos en el pensar argentino decimonónico." Cuyo. Anuario de Filosofía Argentina y Americana 26, pp. 61-100.

LAFARGA, Francisco. (2009) "Zozaya, Antonio". En: Lafarga, Francisco \& Luis Pegenaute (eds.) 2009. Diccionario histórico de la traducción en España. Madrid: Gredos, pp. 1190-1191.

LARraz ElorriagA, Fernando (2011) "Los exiliados y la colecciones editoriales en Argentina (1938-1953)." En: Pagni, Andrea (ed.) 2011. El exilio republicano español en México y Argentina. Historia cultural, instituciones literarias, medio. Madrid: Iberoamericana/Vervuert, pp. 129-144. 
PÉREZ DE LA DEHESA, Rafael. (1969) "La editorial Sempere en Hispanoamérica y España." Revista Iberoamericana 69, pp. 551-555.

PyM, Anthony. (1998) Method in Translation History. Manchester: St Jerome.

RAMOS EsCANDÓn, Carmen. (2001) "Genaro García: historiador feminista de fin de siglo." Signos Históricos 5, pp. 87-107.

Romero, Luis Alberto. (1990) "Buenos Aires en la entreguerra: libros baratos y cultura de los sectores populares." En: Armus, Diego (ed.) 1990. Mundo urbano y cultura popular. Buenos Aires: Editorial Sudamericana, pp. 39-69.

SÁnChEZ Ortíz de URBinA, Ricardo (1966) "Krausismo". En: Varios autores. 1966. Enciclopedia de la Cultura Española. Madrid: Editora Nacional, Proyecto filosofía en español. Versión electrónica: <http: //www. filosofia. org/enc/ece/ e30825.htm>

SAntoyo, Julio César (2009) "Darwin, Charles Robert." En: Lafarga, Francisco \& Luis Pegenaute (eds.) 2009. Diccionario histórico de la traducción en España. Madrid: Gredos, pp. 286-287.

SAPIRO, Gisèle. (2008) Translatio: le marché de la traduction en France à l'heure de la mondialisation. Paris: CNRS.

SORÁ, Gustavo. (2009) "Des éclats du siècle: unité et désintégration dans l'éditions hispano-américaine en sciences sociales.” En: Sapiro, Gisèle (ed.) 2009. Les contradictions de la mondialisation éditoriale. Paris: Nouveau Monde, pp. 93-116.

Soriano Nieto, Nieves. (2009) "Comte, Auguste." En: Lafarga, Francisco \& Luis Pegenaute (eds.) 2009. Diccionario histórico de la traducción en España. Madrid: Gredos, pp. 251-252.

Subercaseaux, Bernardo. (2004) Historia de las ideas y de la cultura en Chile. III: El centenario y las vanguardias. Santiago de Chile: Editorial Universitaria.

Willson, Patricia. (2004) La constelación del Sur. Traductores y traducciones en la literatura argentina del siglo XX. Buenos Aires: Siglo XXI.

Woodward JR., Ralph Lee (ed.) (1971) Positivism in Latin America, 1850-1900. Are Order and Progress Reconcilable? Lexington: D. C. Heath and Company.

ZEA, Leopoldo (ed.) (1980) Pensamiento positivista latinoamericano. Caracas: Biblioteca Ayacucho. 


\section{NOTAS BIOGRÁFICAS / BIONOTES}

\section{Nayelli Castro}

Nayelli Castro obtuvo un doctorado en Estudios de Traducción en la Universidad de Ottawa en 2012. Se interesa, entre otros temas, por la historia y la sociología de la traducción. En su tesis doctoral, Regards sociologiques sur la traduction philosophique, Mexique (1940-1970), estudia la traducción de filosofía al español desde una perspectiva sociológica. Actualmente realiza una estancia postdoctoral en El Colegio de México.

Nayelli Castro completed her PhD in Translation Studies at the University of Ottawa in 2012. Her research interests include the history and sociology of translation. In her doctoral thesis, Regards sociologiques sur la traduction philosophique, Mexique (1940-1970), she studies the translation of philosophy into Spanish from a sociological perspective. Currently, she is a postdoctoral researcher at El Colegio de México.

\section{Clara Foz}

Profesora de traducción en la School of Translation and Interpretation de la Universidad de Ottawa. Doctora en Traductología por la ESIT de la Universidad de París III. Traductora jurada. Sus campos de investigación son la historia de la traducción y la metodología de la investigación en Estudios de Traducción.

Professor at the School of Translation and Interpretation of the University of Ottawa. She holds a doctorate in Translation Studies from ESIT -Université Paris III-. Sworn translator. Her research interests include history of translation and methodology of research in Translation Studies. 